\title{
Surface areas of altitudinal zones on sub-Antarctic Marion Island
}

\author{
K. I. Meiklejohn ${ }^{1}$ and V. R. Smith ${ }^{2}$ \\ (1) Department of Geography, Geoinformatics and Meteorology, University of \\ Pretoria, Pretoria, 0002, South Africa \\ (2) Department of Botany and Zoology, Stellenbosch University, Private Bag X1, \\ Matieland, 7602, South Africa
}

\author{
K. I. Meiklejohn \\ Email: ian.meiklejohn@up.ac.za
}

\section{Without Abstract}

French and Smith (1985) used climatic, edaphic and vegetation information in an ordinal comparison to define the position of sub-Antarctic islands in the "tundra spectrum". They showed that sub-Antarctic islands possess almost the full range of vegetation types found in Northern Hemisphere tundra. A particularly significant finding concerned how altitude influenced the islands' climatic and edaphic regimes and their vegetation:

"The overall position of the sub-Antarctic islands in the tundra spectrum can be expressed by the altitudinal change needed to go from the lowest altitude "tundra vegetation", usually meadow or mire, to fellfield (or equivalent)-i.e. the increase in altitude required for the elimination of all other vegetation types. In the Northern Hemisphere sites, this is 1-2 $\mathrm{m}$ in the high Arctic, 2-20 $\mathrm{m}$ in low-Arctic and more severe Sub-arctic sites, 20-200 $\mathrm{m}$ in the milder Sub-arctic/Sub-alpine areas, and up to 1,000 m or more in cool-temperate and temperate alpine zones. In the southern islands, the equivalent vegetation change takes 5-50 m, while a further change of 50-500 m eliminates all plants, except scattered short grasses and cushion-forming forbs, and cryptogams" (French and Smith 1985).

Thus, altitude strongly influences vegetation and soils at sub-Antarctic islands, as much as it does at the more climatically extreme Northern Hemisphere tundras that occur at 20 $30^{\circ}$ higher latitudes than any of the islands.

On Marion Island $\left(47^{\circ} \mathrm{S}, 38^{\circ} \mathrm{E}\right)$, altitude is a particularly important determinant of many of the abiotic and biotic components of the ecosystem; e.g. the composition and nutrient status of the island's terrestrial habitats (Smith et al. 2001), distribution and densities of the soil fauna (Barendse et al. 2002), densities and activities of soil microbial populations (French and Smith 1986; Grobler et al. 1987), and functional processes such as decomposition (Smith et al. 1993) and soil respiration rate (Smith 2003). The frequency 
of diurnal freeze-thaw events in the soil and the depth of freezing both increase sharply with altitude (Boelhouwers et al. 2003) and are crucial to soil-atmosphere energy exchange, trophodynamics and nutrient cycling. Research at the island is increasingly focussing on incorporating all this structural and functional information into models and we anticipate that the models will need altitudinal spatial information. Here, we provide estimates of the areas occupied in 20 and $100 \mathrm{~m}$ altitudinal bands on the island.

Projected surface areas and actual surface areas were calculated using a $20 \mathrm{~m}$ resolution digital elevation model (DEM), developed from data supplied by the Chief Directorate: Surveys and Mapping, Mowbray, South Africa. Vector contours (10 m intervals), spot heights and drainage lines were used to interpolate the $20 \mathrm{~m}$ DEM using the "Topo to Raster" module in ArcGIS ${ }^{\circledR}\left(\operatorname{ArcInfo}^{\circledR}\right) .{ }^{1}$ The DEM was analysed using the Spatial Analyst ${ }^{\circledR}$ extension of ArcGIS ${ }^{\circledR} .{ }^{1}$ The DEM was then classified into $20 \mathrm{~m}$ altitudinal bands and the projected surface area (i.e. planimetric area, based on a Transverse Mercator projection) calculated from the number of raster pixels in each band and the pixel resolution $(20 \mathrm{~m} \times 20 \mathrm{~m})$. Actual surface areas (the ground surface area) were calculated using the raster-based method of Jenness (2004). The results are presented in Table 1.

Table 1 Projected and actual surface areas in 20 and $100 \mathrm{~m}$ altitudinal bands on Marion Island

\begin{tabular}{|c|c|c|c|c|c|}
\hline Altitude (m) & $\begin{array}{l}\text { Projected } \\
\text { area }\left(\mathbf{m}^{2}\right)\end{array}$ & $\begin{array}{l}\text { Surface area } \\
\left(\mathbf{m}^{2}\right)\end{array}$ & Altitude (m) & $\begin{array}{l}\text { Projected } \\
\text { area }\left(\mathbf{m}^{2}\right)\end{array}$ & $\begin{array}{l}\text { Surface area } \\
\left(\mathbf{m}^{2}\right)\end{array}$ \\
\hline $0-20$ & $6,754,800$ & $6,867,700$ & $0-100$ & $67,653,200$ & $68,713,900$ \\
\hline $20-40$ & $17,334,000$ & $17,543,900$ & $100-200$ & $39,524,400$ & $40,914,440$ \\
\hline $40-60$ & $18,920,400$ & $19,158,400$ & $200-300$ & $34,694,800$ & $35,975,930$ \\
\hline $60-80$ & $14,223,600$ & $14,457,000$ & $300-400$ & $30,002,800$ & $31,082,480$ \\
\hline $80-100$ & $10,420,400$ & $10,686,900$ & $400-500$ & $27,155,200$ & $28,147,240$ \\
\hline $100-120$ & $8,566,400$ & $8,848,660$ & $500-600$ & $22,970,800$ & $23,915,090$ \\
\hline $120-140$ & $8,091,200$ & $8,365,930$ & $600-700$ & $20,347,200$ & $21,209,590$ \\
\hline $140-260$ & $8,142,000$ & $8,428,850$ & $700-800$ & $15,372,400$ & $16,118,600$ \\
\hline $160-180$ & $7,144,000$ & $7,416,030$ & $800-900$ & $12,128,000$ & $12,713,060$ \\
\hline $180-200$ & $7,580,800$ & $7,854,970$ & $900-1,000$ & $9,397,600$ & $9,889,450$ \\
\hline $200-220$ & $7,542,400$ & $7,805,770$ & $1,000-1,100$ & $7,201,600$ & $7,610,330$ \\
\hline $220-240$ & $6,972,400$ & $7,229,290$ & $1,100-1,200$ & $3,696,000$ & $3,930,286$ \\
\hline $240-260$ & $6,858,000$ & $7,112,790$ & $>1,200$ & 181,600 & 197,220 \\
\hline $260-280$ & $6,702,400$ & $6,954,390$ & & & \\
\hline $280-300$ & $6,619,600$ & $6,873,690$ & & & \\
\hline $300-320$ & $6,075,200$ & $6,314,580$ & & & \\
\hline $320-340$ & $6,290,800$ & $6,513,340$ & & & \\
\hline
\end{tabular}




\begin{tabular}{|c|c|c|c|c|c|}
\hline Altitude (m) & $\begin{array}{l}\text { Projected } \\
\text { area }\left(\mathbf{m}^{2}\right)\end{array}$ & $\begin{array}{l}\text { Surface area } \\
\left(\mathbf{m}^{2}\right)\end{array}$ & Altitude (m) & $\begin{array}{l}\text { Projected } \\
\text { area }\left(\mathbf{m}^{2}\right)\end{array}$ & $\begin{array}{l}\text { Surface area } \\
\left(\mathbf{m}^{2}\right)\end{array}$ \\
\hline $340-360$ & $6,525,200$ & $6,732,920$ & & & \\
\hline $360-380$ & $5,802,000$ & $6,005,340$ & & & \\
\hline $380-400$ & $5,309,600$ & $5,516,300$ & & & \\
\hline $400-420$ & $5,933,600$ & $6,137,650$ & & & \\
\hline $420-440$ & $6,282,800$ & $6,483,160$ & & & \\
\hline $440-460$ & $5,394,000$ & $5,594,830$ & & & \\
\hline $460-480$ & $5,041,600$ & $5,237,590$ & & & \\
\hline $480-500$ & $4,503,200$ & $4,694,010$ & & & \\
\hline $500-520$ & $4,769,200$ & $4,951,830$ & & & \\
\hline $520-540$ & $4,499,200$ & $4,685,660$ & & & \\
\hline $540-560$ & $4,884,400$ & $5,074,720$ & & & \\
\hline $560-580$ & $4,485,600$ & $4,680,200$ & & & \\
\hline $580-600$ & $4,332,400$ & $4,522,680$ & & & \\
\hline $600-620$ & $4,332,400$ & $4,510,560$ & & & \\
\hline $620-640$ & $4,512,400$ & $4,687,980$ & & & \\
\hline 640-660 & $4,151,200$ & $4,323,910$ & & & \\
\hline $660-680$ & $3,681,600$ & $3,854,120$ & & & \\
\hline $680-700$ & $3,669,600$ & $3,833,020$ & & & \\
\hline $700-720$ & $3,434,400$ & $3,596,430$ & & & \\
\hline $720-740$ & $3,312,400$ & $3,466,610$ & & & \\
\hline $740-760$ & $2,938,800$ & $3,087,580$ & & & \\
\hline $760-780$ & $2,955,600$ & $3,098,800$ & & & \\
\hline $780-800$ & $2,731,200$ & $2,869,180$ & & & \\
\hline $800-820$ & $2,945,600$ & $3,074,190$ & & & \\
\hline $820-840$ & $2,682,800$ & $2,803,600$ & & & \\
\hline $840-860$ & $2,408,000$ & $2,524,110$ & & & \\
\hline $860-880$ & $2,187,200$ & $2,296,050$ & & & \\
\hline $880-900$ & $1,904,400$ & $2,015,110$ & & & \\
\hline 900-920 & $2,001,600$ & $2,107,790$ & & & \\
\hline $920-940$ & $1,880,800$ & $1,986,780$ & & & \\
\hline $940-960$ & $1,802,400$ & $1,903,450$ & & & \\
\hline 960-980 & $2,116,000$ & $2,207,450$ & & & \\
\hline $980-1,000$ & $1,596,800$ & $1,683,980$ & & & \\
\hline
\end{tabular}




\begin{tabular}{|l|l|l|l|l|l|}
\hline Altitude $(\mathbf{m})$ & $\begin{array}{l}\text { Projected } \\
\left.\text { area } \mathbf{( m}^{\mathbf{2}}\right)\end{array}$ & $\begin{array}{l}\text { Surface area } \\
\left(\mathbf{m}^{\mathbf{2}}\right)\end{array}$ & Altitude $\mathbf{( m )}$ & $\begin{array}{l}\text { Projected } \\
\left.\text { area } \mathbf{( m}^{\mathbf{2}}\right)\end{array}$ & $\begin{array}{l}\text { Surface area } \\
\left(\mathbf{m}^{\mathbf{2}} \mathbf{)}\right.\end{array}$ \\
\hline $1,000-1,020$ & $1,466,000$ & $1,555,350$ & & & \\
\hline $1,020-1,040$ & $1,680,400$ & $1,769,590$ & & & \\
\hline $1,040-1,060$ & $1,357,600$ & $1,440,190$ & & & \\
\hline $1,060-1,080$ & $1,410,000$ & $1,484,510$ & & & \\
\hline $1,080-1,100$ & $1,287,600$ & $1,360,690$ & & & \\
\hline $1,100-1,120$ & $1,104,000$ & $1,162,540$ & & & \\
\hline $1,120-1,140$ & 916,400 & 966,205 & & & \\
\hline $1,140-1,160$ & 806,000 & 857,787 & & & \\
\hline $1,160-1,180$ & 578,000 & 621,689 & & & \\
\hline $1,180-1,200$ & 291,600 & 322,065 & & & \\
\hline $1,200-1,220$ & 152,800 & 165,925 & & & \\
\hline $1,220-1,240$ & 28,800 & 31,295 & & & \\
\hline Total island & $290,325,600$ & $300,417,616$ & & & \\
\hline
\end{tabular}

\section{References}

Barendse J, Mercer RD, Marshall DJ, Chown SL (2002) Habitat specificity of mites on sub-Antarctic Marion Island. Environ Entomol 31:612-625

Boelhouwers J, Holness S, Sumner P (2003) The maritime Subantarctic: a distinct periglacial environment. Geomorphology 52:39-55

French DD, Smith VR (1985) A comparison between Northern and Southern Hemisphere tundras and related ecosystems. Polar Biol 5:5-21

French DD, Smith VR (1986) Bacterial populations in soils of a sub-Antarctic Island. Polar Biol 6:75-82

Grobler DC, Toerien DF, Smith VR (1987) Bacterial activity in soils of a sub-Antarctic Island. Soil Biol Biochem 19:485-490

Jenness JS (2004) Calculating landscape surface area from digital elevation models. Wildl Soc Bull 32:829-839

$<$ Occurrence Type="DOI" $><$ Handle $>10.2193 / 0091-$

7648(2004)032[0829:CLSAFD]2.0.CO;2</Handle></Occurrence $>$ 
Smith VR (2003) Soil respiration and its determinants on a sub-Antarctic island. Soil Biol Biochem 35:77-91

Smith VR, Steenkamp M, French DD (1993) Soil decomposition potential in relation to environmental factors on Marion Island (sub-Antarctic). Soil Biol Biochem 25:16191633

Smith VR, Steenkamp M, Gremmen NJM (2001) Terrestrial habitats on sub-Antarctic Marion Island: their vegetation, edaphic attributes, distribution and response to climate change. S Afr J Bot 67:641-654

\section{Footnotes}

${ }^{1}$ ArcGIS, ArcInfo and Spatial Analysis are registered trademarks of Environmental Systems research Institute, Inc., Redlands, CA, USA. 